\section{Effects on Varying Intravenous Lipid Emulsions on the Small Bowel Epithelium in a Mouse Model of Parenteral Nutrition}

\author{
Yongjia Feng, MD, PhD; Pele Browner, BS; and Daniel H. Teitelbaum, MD
}

Journal of Parenteral and Enteral

Nutrition

Volume 37 Number 6

November 2013 775-786

(C) 2013 American Society

for Parenteral and Enteral Nutrition DOI: $10.1177 / 0148607113491608$

jpen.sagepub.com

hosted at

online.sagepub.com

@SAGE

\begin{abstract}
Background: Injectable fat emulsions (FEs) are a clinically dependable source of essential fatty acids (FA). $\omega-6$ FA is associated with an inflammatory response. Medium-chain triglycerides (MCT, $\omega-3$ FA), fish oil, and olive oil are reported to decrease the inflammatory response. However, the effect of these lipids on the gastrointestinal tract has not been well studied. To address this, we used a mouse model of parenteral nutrition (PN) and hypothesized that a decrease in intestinal inflammation would be seen when either fish oil and MCT or olive oil were added. Methods: Three FEs were studied in adult C57BL/6 mice via intravenous cannulation: standard soybeanbased FE (SBFE), 80\% olive oil -supplemented FE (OOFE), or a combination of a soybean oil, MCT, olive oil, and fish oil emulsion (SMOF). PN was given for 7 days, small bowel mucosa-derived cytokines, animal survival rate, epithelial cell (EC) proliferation and apoptosis rates, intestinal barrier function and mucosal FA composition were analyzed. Results: Compared to the SBFE and SMOF groups, the best survival, highest EC proliferation and lowest EC apoptosis rates were observed in the OOFE group; and associated with the lowest levels of tumor necrosis factor- $\alpha$, interleukin- 6 , and interleukin- $1 \beta$ expression. Jejunal FA content showed higher levels of eicosapentaenoic and docosapentaenoic acid in the SMOF group and the highest arachidonic acid in the OOFE group. Conclusion: The study showed that PN containing OOFE had beneficial effects to small bowel health and animal survival. Further investigation may help to enhance bowel integrity in patients restricted to PN. (JPEN J Parenter Enteral Nutr. 2013;37:775-786)
\end{abstract}

\title{
Keywords
}

intravenous lipids; parenteral nutrition; epithelial cell apoptosis; epithelial cell proliferation; $\omega$-3 lipids; $\omega$ - 6 lipids; medium-chain triglycerides; fish oil

Intravenous (IV) lipids should comprise between $30 \%$ and $50 \%$ of all nonnitrogen calories. However, the refinement and development of IV fat emulsions (IVFEs) have been complex. ${ }^{1}$ All IVFEs provided in the United States are derived from soybean oil and are predominately long-chain triglyceride (LCT) $\omega-6$ polyunsaturated fatty acids (FAs) and thus, in certain settings, have been shown to drive a proinflammatory response. To address these potential shortcomings, other sources of fat emulsions (FEs), including medium-chain triglycerides (MCTs), can reduce the total amount of LCTs delivered to the patient. Additional advantages of MCTs include greater solubilization of the lipid component, decreased accumulation of lipids in the liver, and improved clearance and reduced production of proinflammatory mediators. ${ }^{2,3}$ In addition, use of MCTs may actually improve immune function by decreasing the levels of tumor necrosis factor- $\alpha(\mathrm{TNF}-\alpha){ }^{4}$ Another strategy is the replacement of soybean-predominant FE with an olive oilpredominant fat. Use of agents with $80 \%$ olive oil can reduce a patient's exposure to $\omega-6$ FAs. Furthermore, olive oils contain a blend of $\omega-3, \omega-6$, and $\omega-9$ FAs and other antioxidants, including polyphenols. Another alternative FE strategy has been to use fish oil. A fish oil FE is composed of almost entirely $\omega-3$ FAs and may also prevent a proinflammatory state. ${ }^{5}$
The ideal IVFE remains elusive, and gaining the ideal balance of FAs may have tremendous implications for patient outcomes, including mortality. ${ }^{6,7}$ Comparisons of FAs have identified that even clinically approved IVFEs can lead to

From the Section of Pediatric Surgery, Department of Surgery, the University of Michigan Medical School and the C. S. Mott Children's Hospital, Ann Arbor, Michigan.

Financial disclosure: The study was supported by a grant from the Baxter Healthcare Corp. Baxter did not have an influence on the contents of this article or preview its contents.

Supplementary material for this article is available on the Journal of Parenteral and Enteral Nutrition website.

Received for publication January 2, 2013; accepted for publication May 6, 2013.

This article originally appeared online on June 11, 2013.

\section{Corresponding Author:}

Yongiia Feng, MD, PhD, Section of Pediatric Surgery, University of Michigan, Mott Children's Hospital, 1540 E. Hospital Dr, SPC 4211, Ann Arbor, MI 48109-4211, USA.

Email: yongfeng@umich.edu. 
essential FA deficiencies, and thus further optimization of formulas is needed. ${ }^{8}$ Use of such alternative FEs has shown promising effects in reducing a proinflammatory injury in the liver. ${ }^{9}$ However, although many of these now commercially available FEs have been studied in terms of their system effects, little is known regarding the action of these fats on the intestinal epithelium. This has significant implications as the administration of parenteral nutrition (PN) results in significant loss of intestinal epithelial barrier function (EBF) and profound intestinal mucosal atrophy ${ }^{10}$; these changes have been associated with a marked increase in the mucosal expression of several proinflammatory cytokines. ${ }^{11}$ However, it is unknown if different IVFEs can influence these adverse changes. To address this, we used a mouse PN model and hypothesized that a decrease in intestinal inflammatory-linked cytokines and intestinal atrophy would be seen when alternative IVFEs were added to existing soybean lipid emulsions, thereby reducing the total exposure to soybean oil.

\section{Methods}

\section{PN Animal Model}

Male specific pathogen-free (SPF) C57BL/6J mice (10-12 weeks old; Jackson Laboratory, Bar Harbor, ME) were maintained under temperature-, humidity-, and light-controlled conditions. Mice were initially fed ad libitum with standard mouse chow and water and allowed to acclimate for 1 week prior to surgery. During the administration of IV solutions, mice were housed in metabolic cages to prevent coprophagia. Studies conformed to guidelines for care established by the University Committee on Use and Care of Animals at the University of Michigan, and protocols were approved by that committee (No. 03986). Methods of cannulation and PN infusion were similar to that previously described. ${ }^{12-14}$ The control group received an IV crystalloid solution (dextrose $5 \%$ in $0.45 \mathrm{NS}$ with $20 \mathrm{mEq} \mathrm{KCl} / \mathrm{L}$ ) at $0.3 \mathrm{~mL} / \mathrm{h}$ and standard laboratory mouse chow. The PN groups received an IV PN solution at 7.2 $\mathrm{mL} / 24$ hours but were allowed water ad libitum. All study groups had complete nutrient supplementation, with all essential vitamins, trace elements, and minerals being supplied; details of caloric delivery have been published in this journal. ${ }^{15}$ All animals received similar daily energy and nitrogen delivery and were euthanized at 7 days using $\mathrm{CO}_{2}$. The small intestine was exposed from the ligament of Treitz. We discarded the first $5 \mathrm{~cm}$ of jejunum and then took $2 \mathrm{~cm}$ of jejunum for FA analysis, $1 \mathrm{~cm}$ of jejunum for RNA extraction, another $2 \mathrm{~cm}$ for histology, and $4 \mathrm{~cm}$ for Ussing chamber testing (Suppl. Fig. S1).

\section{IVFE and Study Masking}

Three IVFEs were studied ( $\mathrm{n}=8$ /group) in adult C57BL/6 mice via IV cannulation: standard soybean-based FE (SBFE), olive oil-supplemented FE (OOFE; composed of $80 \%$ olive oil and $20 \%$ soybean oil), and a combination soybean oil (30\%), medium-chain triglycerides (30\%), olive oil (25\%), and fish oil emulsion (15\%) (SMOF). To ensure a nonbiased approach, the FEs were initially masked so that investigators did not know (ie, were blinded) the identity of the FEs until the data analysis was completed. These 3 groups were compared with a sham or chow-fed group of mice. All FEs were derived from commercial products as follows: SBFE (Intralipid; Fresenius Kabi, Uppsala, Sweden), OOFE (Clinoleic; Baxter Healthcare, Deerfield, IL), and SMOF (SMOFlipid, Fresenius Kabi). Each FE was studied at 3 concentrations, comprising $10 \%, 20 \%$, or $30 \%$ of total daily energy delivery as fat. Compensation of energy delivery between groups was provided by the addition or subtraction of dextrose to ensure each group was isocaloric, isonitrogenous, and isovolemic. All volumes, energy delivery, and nitrogen concentrations were balanced between all study groups. Furthermore, all solutions were compounded by a sterile, class 100,000 facility at our university (HomeMed Infusion Company) as a 3:1 solution.

\section{Intestinal Morphology Assessment}

Villus height and crypt depth were measured in at least 20 well-oriented full-length crypt-villus units per specimen and averaged. Data were analyzed using commercially available digital image analysis software NIS-Elements, AR 3.0 (Nikon Instruments Inc, Melville, NY).

\section{Epithelial Cell Proliferation and Apoptosis}

For epithelial cell (EC) proliferation, immunofluorescent staining for proliferating cell nuclear antigen (PCNA) was performed as previously described. ${ }^{16}$ Results are expressed as the ratio of the number of PCNA-positive cells to the total number of crypt cells. A total of 15-20 crypts were measured for each mouse. EC proliferation was also measured with BrdU staining, as previously described. ${ }^{17}$ Immunofluorescent staining for active caspase- 3 was performed to assess EC apoptosis, as previously described. ${ }^{10}$ Results are expressed as an apoptotic index (\% active caspase-3-positive cell number/villi cell number, using a mean of $10-15$ villi/mouse).

\section{Real-Time Polymerase Chain Reaction}

RNA extraction of jejunum mucosal scrapings was processed as previously described. ${ }^{10}$ Oligomers were designed using an optimization program (www.premierBiosoft.com). Real-time polymerase chain reaction was performed and normalized to $\beta$-actin. ${ }^{18}$

\section{Immunofluorescence Microscopy}

A $0.5-\mathrm{cm}$ section of fresh jejunum $(\sim 10 \mathrm{~cm}$ from the ligament of Treitz) was harvested and immunofluorescence staining was 
performed as previously described. ${ }^{19}$ Fluorescence was analyzed by using an Olympus BX-51 upright light and fluorescence microscope (Olympus, Center Valley, PA), and images were stacked using Nikon image software (Nikon, Tokyo, Japan) and processed using Adobe Photoshop CS4 (Adobe, San Jose, CA).

\section{Epithelial Barrier Function}

Full-thickness jejunal tissue was mounted in Ussing chambers. Assessment of EBF was measured using previously described methods. ${ }^{11}$ Jejunum transepithelial resistance (TER) was determined by measurements of spontaneous voltage and isoelectric current using the Ohms law after a 30-minute equilibration period and was expressed $\Omega \cdot \mathrm{cm}^{2}$.

\section{FA Analysis}

After mice jejunum tissue, which had been frozen at $-80^{\circ} \mathrm{C}$, was slowly warmed, a 150 -g piece was cut and transferred to a blood collection tube $(100 \times 16 \mathrm{~mm})$. The weight $(\mathrm{mg})$ of the tissue piece was recorded and then put on ice. Then, $1.0 \mathrm{~mL}$ of cooled phosphate-buffered saline was added to the tube, and the sample was homogenized with an Omni homogenizer and Omni-Tips (Omni international homogenizer company, Kennesaw, GA) for 30 minutes and then put back on ice. Next, $100 \mu \mathrm{L}$ of jejunum homogenate was transferred to a disposable glass tube $(16 \times 100 \mathrm{~mm}$; Pyrex with Teflon-lined screw caps). To these tubes, $20 \mu \mathrm{L}$ of tricosanoic acid (C23:0, $0.989 \mathrm{mg} /$ $\mathrm{mL}$ ) as an internal standard and $2000 \mu \mathrm{L}$ of methanol-benzene $4: 1(\mathrm{v} / \mathrm{v})$ were then added. The tubes were vortexed gently. Tubes were placed in a dry ice bath for 10 minutes. Then, 250 $\mu \mathrm{L}$ of acetyl chloride was added to each tube while on dry ice, the caps were tightly closed, and the tube rack was transferred at room temperature (RT) until the reaction mixture had melted. The tubes were then vortexed gently. The tubes were purged with nitrogen gas, with the Teflon-lined caps tightly closed again, and subjected to RT for 24 hours. The tubes were cooled in an ice bucket, and then $5 \mathrm{~mL}$ of $6 \% \mathrm{~K}_{2} \mathrm{CO}_{3}$ solution was added slowly to stop the reaction and neutralize the mixture. The reaction mixture was vortexed and then centrifuged at $3000 \mathrm{rmp}$ for 20 minutes $\left(25^{\circ} \mathrm{C}\right)$ to separate layers. An aliquot $(150-200 \mu \mathrm{L})$ was removed by gently tilting the tubes and drawing off the benzene layer with a hand pipettor, using the long-tailed pipet tips. The contents were transferred in the sample vials (amber color with glass inserts) for gas chromatography (GC) analysis. For each sample, peaks were identified based on the retention time compared with the corresponding reference standards; peak area was determined for each FA methyl ester, including C23:0 methyl ester (as internal standard), from the GC chromatograph. ${ }^{20}$ FA methyl ester concentrations in the sample were normalized and calculated relative to that of the internal standard (C23:0) and tissue amount.

\section{Data Analysis}

Data were expressed as mean \pm standard deviation (SD). Statistical analysis employed paired $t$ tests for comparison of 2 means and a 1-way analysis of variance (ANOVA) for comparison of multiple groups (with a Bonferroni post hoc analysis to assess statistical differences between groups), and 2-way ANOVA test was used for categorical data (Prism software; GraphPad Software, San Diego, CA). Statistical significance was defined as $P<.05$.

\section{Results}

Preliminary studies were performed with FE concentrations being studied at $10 \%, 20 \%$, and $30 \%$. Due to the complexity of reporting the results, we believed that the most clinically relevant percentage that would report the bulk of the results was $30 \%$ of total daily calories as fat, as this approximates a typical level added to PN solutions. As well, this percentage was most similar to that previously studied by our research group. Additional results at the $10 \%$ and $20 \% \mathrm{FE}$ concentrations are reported in Table 1.

\section{Survival and Body Weight}

Survival rates were compared with mice in each group (Figure 1A). Sham mice had a $100 \%$ survival after the 7 -day study period. Distinct differences in survival were noted between the FE groups. Mice in the SBFE group had an approximately $65 \%$ survival, which was similar to our previously reported rate of $70 \%{ }^{16,21,22}$ Mice in the SMOF group, although not statistically different from the SBFE group, had a lower survival rate $(55 \%)$. Interestingly, mice in the OOFE group had the highest survival rate $(100 \%)$, and rates were identical to the $100 \%$ survival of the chow-fed controls.

Body weight loss occurred in all PN mice, as has been reported previously (Figure 1B) ${ }^{10,21}$ The range of weight loss was $4-5 \mathrm{~g}$ over the 1 -week infusion period, and although significantly greater than sham (chow-fed) mice, no differences were identified between the FE groups.

\section{Intestinal Morphology and Epithelial Cell Turnover}

As previously reported, administration of PN led to a significant degree of intestinal atrophy. Table 1 shows that villus height decreased significantly in all PN-treated groups compared with the sham group. The greatest decline was found in the SBFE group. Although lower than the sham group, the SMOF and OOFE groups both had longer villus lengths compared with the SBFE group. No significant differences were seen in the crypt depth between the study groups.

Since PN-associated villus atrophy is due to a loss of EC proliferation and an increase in EC apoptosis, ${ }^{23,24}$ we 
Table 1. Summary of Key Clinical and Cytokine Data.

\begin{tabular}{lcccccccccc}
\hline Group & Sham & SBFE 10\% & SMOF 10\% & OOFE 10\% & SBFE 20\% & SMOF 20\% & OOFE 20\% & SBFE 30\% & SMOF 30\% & OOFE 30\% \\
\hline Crypt depth & $22.2 \pm 1.9$ & $23.5 \pm 1.1$ & $24.1 \pm 0.6$ & $23.5 \pm 1.1$ & $22.8 \pm 0.5$ & $17.2 \pm 1.7$ & $28.6 \pm 1.5$ & $24.9 \pm 1.3$ & $16.3 \pm 0.8$ & $25.1 \pm 2.1$ \\
Villus height & $251.3 \pm 74$ & $160.9 \pm 18.0$ & $218.8 \pm 32.4$ & $196.3 \pm 22.9$ & $206.1 \pm 51.5$ & $192.2 \pm 5.5$ & $189.0 \pm 14.9$ & $165.7 \pm 16.7$ & $168.5 \pm 23.1$ & $183.0 \pm 14.7$ \\
PCNA & $0.42 \pm 0.03$ & $0.14 \pm 0.03$ & $0.12 \pm 0.03$ & $0.24 \pm 0.04$ & $0.24 \pm 0.02$ & $0.25 \pm 0.05$ & $0.25 \pm 0.05$ & $0.22 \pm 0.04$ & $0.24 \pm 0.05$ & $0.28 \pm 0.02$ \\
TNF- $\alpha^{\mathrm{a}}$ & $0.30 \pm 0.07$ & $0.49 \pm 0.30$ & $0.95 \pm 0.79$ & $0.44 \pm 0.21$ & $0.42 \pm 0.22$ & $0.38 \pm 0.12$ & $0.19 \pm 0.08$ & $0.42 \pm 0.15$ & $0.69 \pm 0.16$ & $0.34 \pm 0.20$ \\
TNF-R1 $^{\mathrm{a}}$ & $67.0 \pm 12.8$ & $96.4 \pm 18.8$ & $116.6 \pm 36.5$ & $73.0 \pm 22.1$ & $111.9 \pm 29.6$ & $74.8 \pm 11.6$ & $52.3 \pm 21.5$ & $134.7 \pm 39.6$ & $79.5 \pm 18.7$ & $43.6 \pm 6.2$ \\
IL-1 $^{\mathrm{a}}$ & $3.37 \pm 2.22$ & $4.84 \pm 3.89$ & $3.89 \pm 2.60$ & $2.46 \pm 1.44$ & $6.08 \pm 4.28$ & $2.39 \pm 2.48$ & $1.30 \pm 1.03$ & $5.87 \pm 3.04$ & $2.66 \pm 1.49$ & $1.67 \pm 1.37$ \\
IL-6 $^{\mathrm{a}}$ & $0.25 \pm 0.12$ & $0.30 \pm 0.23$ & $0.73 \pm 0.25$ & $0.16 \pm 0.16$ & $0.26 \pm 0.15$ & $0.22 \pm 0.09$ & $0.15 \pm 0.07$ & $0.45 \pm 0.23$ & $0.11 \pm 0.11$ & $0.09 \pm 0.04$ \\
INF- $^{\mathrm{a}}$ & $0.19 \pm 0.09$ & $0.51 \pm 0.03$ & $1.33 \pm 0.93$ & $0.43 \pm 0.35$ & $0.14 \pm 0.05$ & $0.60 \pm 0.29$ & $0.18 \pm 0.05$ & $0.26 \pm 0.19$ & $0.26 \pm 0.11$ & $0.22 \pm 0.15$ \\
Tlr4 $^{\mathrm{a}}$ & $0.59 \pm 0.09$ & $1.01 \pm 0.30$ & $1.41 \pm 0.90$ & $0.43 \pm 0.20$ & $0.56 \pm 0.28$ & $0.19 \pm 0.09$ & $0.50 \pm 0.33$ & $1.08 \pm 0.36$ & $0.68 \pm 0.23$ & $0.57 \pm 0.32$ \\
\hline
\end{tabular}

IL, interleukin; INF- $\gamma$, interferon $\gamma$; OOFE, $80 \%$ olive oil-supplemented fat emulsion; PCNA, proliferating cell nuclear antigen; SBFE, standard soybean-based fat emulsion; SMOF, combination of soybean oil, medium-chain triglycerides, olive oil, and fish oil emulsion; TNF, tumor necrosis factor. ${ }^{a}$ Multiplied by 1000 .

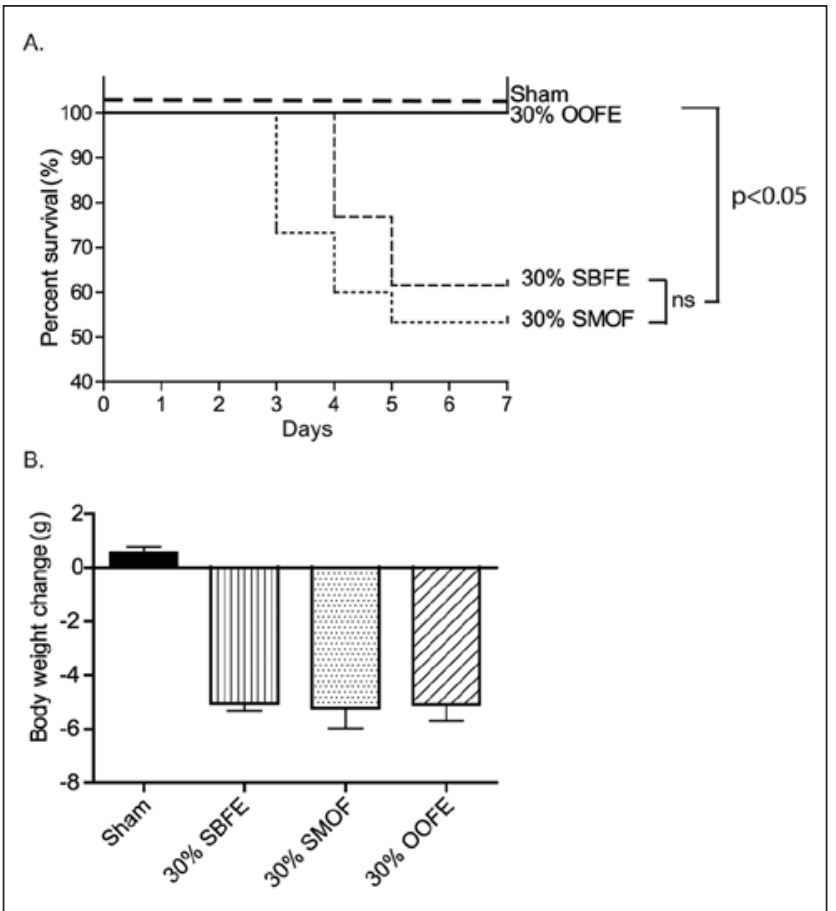

Figure 1. Different fat emulsions significantly affected mouse survival. (A) Date of death was recorded for mice over the 7 days of study. The results are recorded on a survival curve. Of note was a significantly improved survival rate in the $30 \%$ OOFE group (100\% survival) compared with the SBEF and SMOF groups. (B) Body weight was recorded before and after the 1 week of PN administration. No significant differences were noted between the PN groups. OOFE, 80\% olive oil-supplemented fat emulsion; PN, parenteral nutrition; SBFE, standard soybeanbased fat emulsion; SMOF, combination of soybean oil, mediumchain triglycerides, olive oil, and fish oil emulsion.

investigated both. Figure 2 shows EC apoptosis rates, as measured by active caspase- 3 staining in the $30 \%$ FE groups compared with enteral controls (sham). Note that EC apoptosis rates rose significantly (3-fold greater than the sham group) in the SBFE group. Rates of apoptosis were somewhat lower in the SMOF group (not significantly different), and levels declined significantly in the OOFE group to rates similar to those seen in the sham group.

EC proliferation rates were examined using 2 methods, PCNA (Figure 3A) and BrdU (Figure 3B) immunofluorescent staining. Both methods showed similar findings, including a significant decline in EC proliferation rates between all study groups vs shams. However, between the 3 FE groups, differences were seen. Of note, EC proliferation rates were significantly higher in the 30\% OOFE group compared with the SBFE group (for PCNA), and when evaluated by BrdU staining, the EC proliferation rates in OOFE mice were significantly higher compared with both the SBFE and SMOF groups.

To better address the mechanisms that may have led to these changes, several gene factors associated with EC proliferation and apoptosis were examined (Figure 4). Interestingly, despite the SBFE group having the lowest EC proliferation rate and greatest degree of atrophy, messenger RNA (mRNA) expression of EGF, although not significantly different, was actually highest in the SBFE group and lowest in the OOFE group. The EGF receptor, ErbB1, significantly decreased in the SMOF and OOFE groups, and epiregulin and transforming growth factor$\alpha$ (TGF- $\alpha$ ) significantly declined in the OOFE group (Figure $4 C, D)$. Thus, the precise mechanism that led to improved EC proliferation is not clearly identified.

\section{Intestinal Muscosal Gene Expression}

mRNA expression of several proinflammatory factors and junctional proteins was examined in each study group, and distinct differences were noted (Figures 5 and 6). SBFE administration was associated with the upregulation of TNF- $\alpha$, TNF receptor 1 (TNFR1), interleukin (IL)-1 $\beta$, and IL-6. In addition, SBFE was associated with an upregulation of Toll-like receptor 4 (Tlr4). Somewhat surprisingly, although the SMOF group was associated with slightly lower proinflammatory cytokine abundances, SMOF was associated with an increase in TNF- $\alpha$ abundance. In contrast, the OOFE group was associated with a 


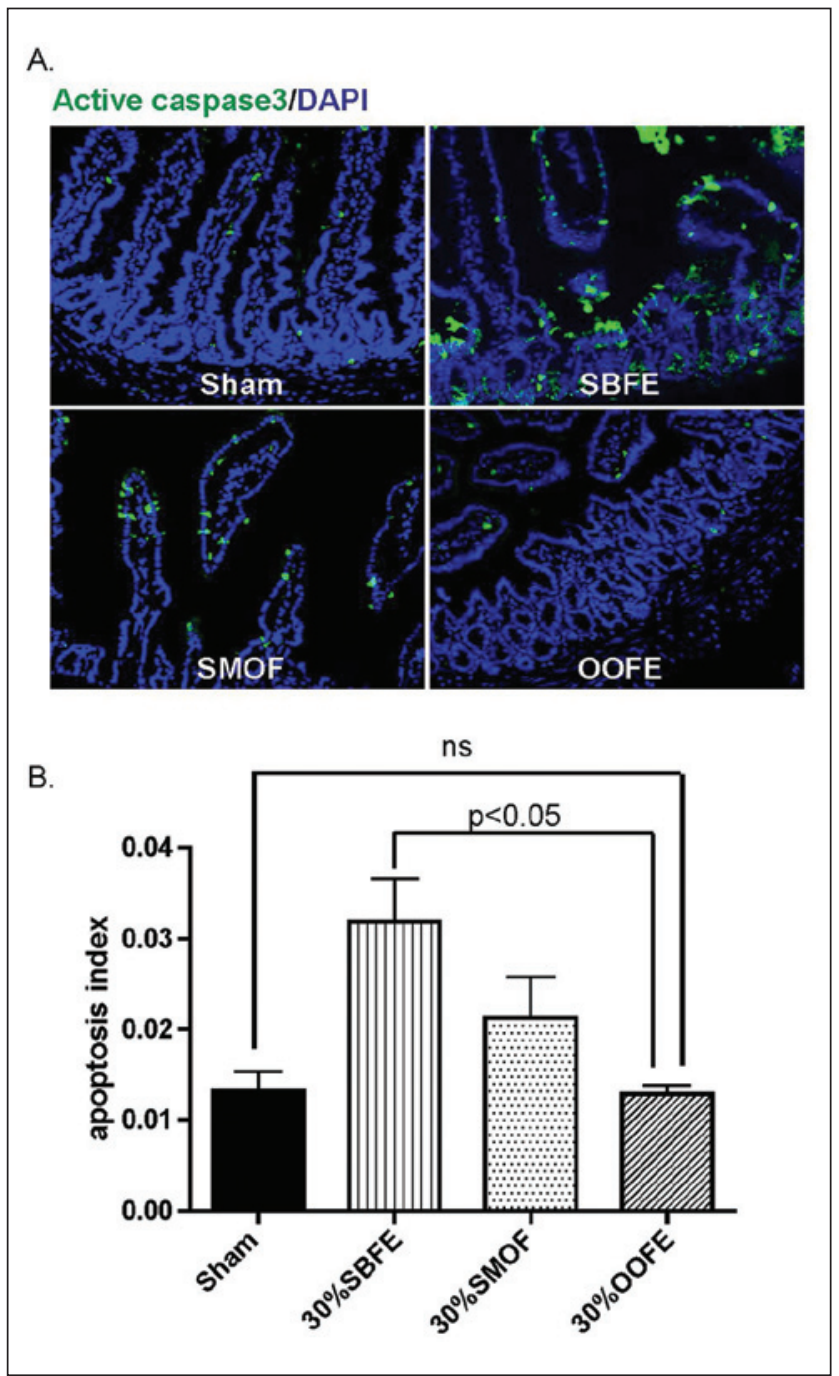

Figure 2. EC apoptosis rates were markedly increased in the SBFE PN group. Apoptosis was measured with active caspase-3 staining in small bowel epithelium. (A) Representative active caspase-3 immunofluorescence images from each study group. (B) Apoptosis index is the ratio of active caspase- 3 cells to the total number of epithelial cells. Note that the SBFE group had the highest EC apoptosis index and OOFE mice had the lowest apoptosis index compared with the SBFE and SMOF groups, with levels reaching those of the sham group. EC, epithelial cell; OOFE, $80 \%$ olive oil-supplemented fat emulsion; PN, parenteral nutrition; SBFE, standard soybean-based fat emulsion; SMOF, combination of soybean oil, medium-chain triglycerides, olive oil, and fish oil emulsion.

significant decline in all proinflammatory cytokines and Tlr4, which were elevated in the SBFE group. No significant changes were noted in the abundance of interferon (INF) $-\gamma$ in any of the treatment groups.

mRNA expression of various tight junction markers was measured (Figure 6A-C). Levels of each tight junction factor varied with the type of FE. Abundance of occludin and ZO-1 levels increased in the SBFE group, with a significant decline in the SMOF and OOFE groups. Similarly, claudin-2 was also increased in the SBFE group (approximately 3-fold higher than the sham group; Figure 6C), as previously reported, ${ }^{21,25}$ and 2-fold higher than sham mice in the SMOF and OOFE groups. Claudin-15, however, was associated with a significant decline in all FE groups (Figure 6C). The overall increase in claudin-2 is consistent with its overall pore-forming action with a resultant loss of barrier function. As well, not shown was a global decrease in claudin-7 abundance in all FE groups.

\section{EBF and Intestinal FA Content}

Barrier function was then measured in Ussing chambers (Figure 6D). Interestingly, despite the increase in occludin and ZO-1, TER results demonstrated a significant decline in levels for all FE groups, and there were no differences noted between the groups.

Finally, tissue was collected to analyze the FA content in proximal intestinal tissue. Table 2 shows the summary of these results at the $30 \% \mathrm{FE}$ level, with total levels essentially the same between the groups. In general, trends followed regardless of the dosage of FE given. The results showed that the sham (enterally fed) group had somewhat higher levels of short-chain FAs (SCFAs) than the SBFE group but similar oleic acid levels. The sham mice had the highest levels of linoleic and linolenic acids and lowest levels of arachidonic acid (AA). Higher levels of eicosapentaenoic acid (EPA) and docosapentaenoic acid (DPA) levels were found in the sham and SMOF groups compared with the SBFF and OOFE groups. Interestingly, the OOFE group exhibited the highest percentage of AA, irrespective of the dosage level.

\section{Discussion}

In this article, we examined 3 commercially available IVFEs regarding their effect on intestinal mucosal gene expression, morphology, epithelial cell turnover, and overall systemic effect on body weight and survival. Interestingly, we found distinct and significant changes between these 3 compounds. Overall weight of the mice was not different between groups, and outcomes at each percentage of FE varied somewhat between groups. However, when focusing the results on the $30 \%$ of energy via the FE group, survival patterns were dramatically different. In particular, use of OOFE resulted in a nearly $100 \%$ survival, whereas SMOF and SBFE resulted in far lower survival rates. Although surprising, other groups have also identified issues with the use of soybean-based lipids. A comparison of survival between fish oil vs soybean FE in a septic rat model demonstrated a significant improvement in survival in the fish oil group. ${ }^{26}$

An area that has not been studied is the effect that FE products might have on intestinal physiology. Although one might suspect that matched energy delivery would suffice to yield 
A.

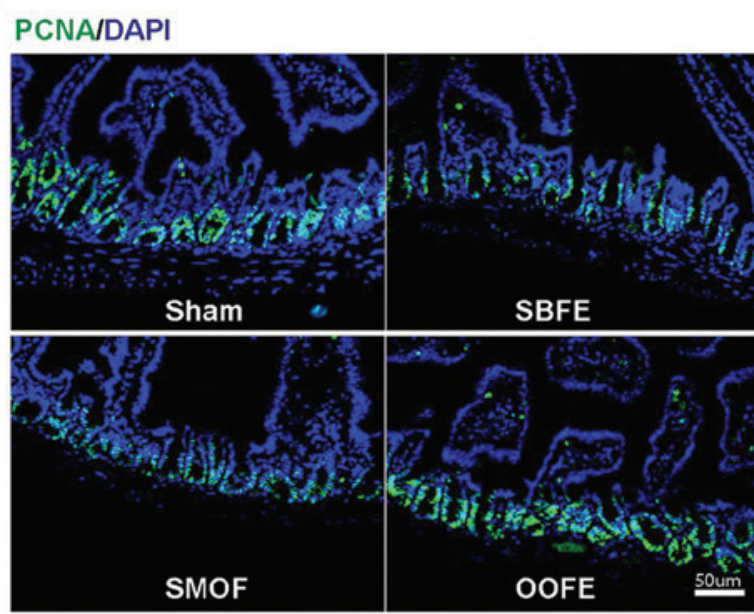

B.

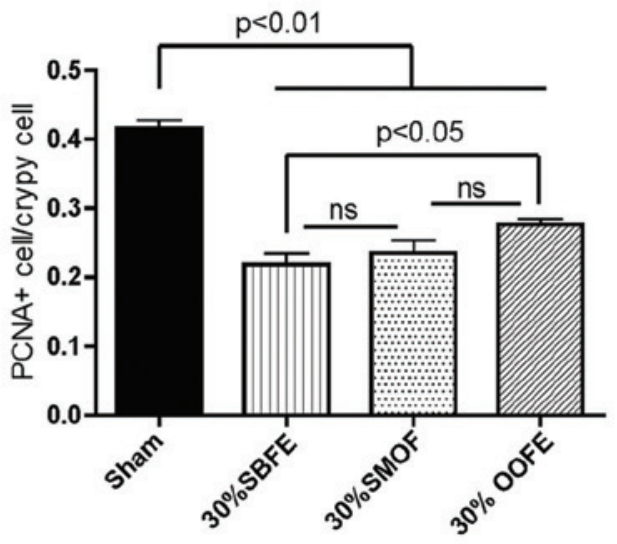

\section{BrdUIDAPI}

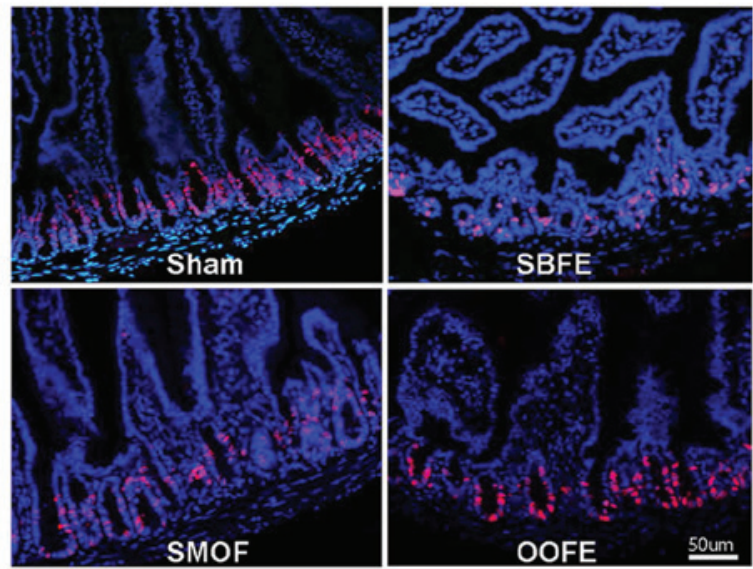

D.

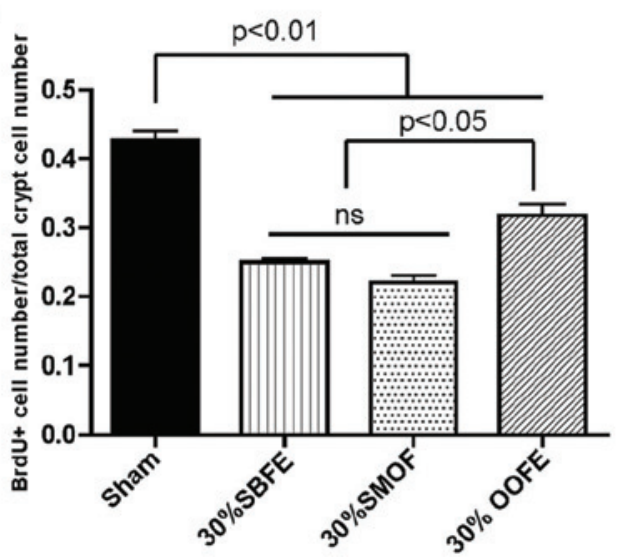

Figure 3. EC proliferation rates in crypt were markedly decreased with PN administration; EC proliferation rates were measured with PCNA and BrdU staining and expressed by the ratio of positive cells numbers compared with the total crypt ECs numbers. (A) Representative images of PCNA staining. (B) Ratio of PCNA-positive cells to total number of crypt cells. Note that all PN groups showed a reduced PCNA expression compared with the sham group, and improved EC PCNA staining was observed in the OOFE group vs SBFE and SMOF groups. BrdU staining (C, D) showed similar results. EC, epithelial cell; OOFE, 80\% olive oil-supplemented fat emulsion; PCNA, proliferating cell nuclear antigen; PN, parenteral nutrition; SBFE, standard soybean-based fat emulsion; SMOF, combination of soybean oil, medium-chain triglycerides, olive oil, and fish oil emulsion.

similar outcomes in each group, striking differences were noted. When examined at the 30\% FE dosing, EC proliferation was significantly higher and EC apoptosis rates were significantly lower in the OOFE group, with a resultant partial prevention of $\mathrm{PN}$-associated mucosal atrophy. mRNA analysis demonstrated several changes in gene expression that appeared somewhat contrary to the EC findings. This included an upregulation of TCF4/LEF transcription factors cyclin D1 and c-Myc in the SBFE group, particularly at the 30\% concentration. In addition, a decline in the stem cell marker LGR5 and declines in cyclin D1 and c-Myc were seen in the OOFE group. It is possible that the upregulation in the SBFE group is a compensatory attempt to prevent the adverse effects (atrophy) occurring with this FE. It may also be an attempt to counter the proinflammatory state associated with the infusion of SBFE.
Our findings showed a trend toward a proinflammatory cytokine state within the intestinal mucosa in mice given SBFE, with reduced levels of these cytokines in the SMOF group and even lower levels in OOFE-treated mice. This included a rise in IL- $1 \beta$ and IL-6. We were surprised to see the increase in TNF- $\alpha$ abundance in the SMOF group. Although this lipid is thought to prevent a proinflammatory state, the etiology of this elevation is unknown, and it is possible that the unique immune system of the gastrointestinal (GI) tract may respond differently to this combination of lipids or may be due to the type of substrate provided to either immunocyte, the upregulation of Tlr4, or the altered intraluminal bacteria. ${ }^{22}$ Although not examined within the GI tract, several studies have pointed to a proinflammatory state with soybean oil. In a study examining fish oil vs soybean oil FE, a significant 


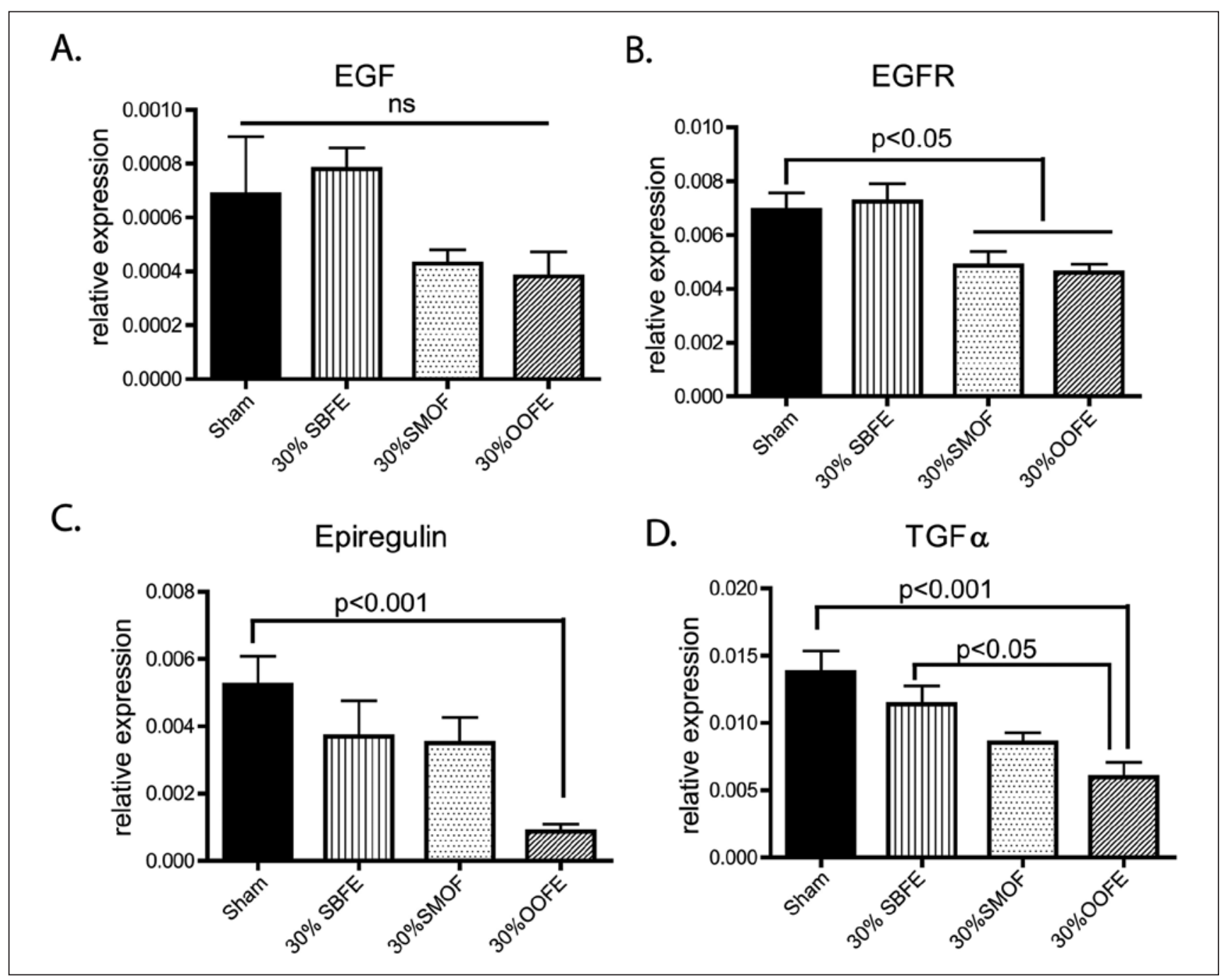

Figure 4. Changes in ErbB ligands and EGF receptor (EGFR) expression with PN administration. Messenger RNA expression of ErbB ligands and the EGFR was determined with real-time polymerase chain reaction, and expressed as a ratio to $\beta$-actin. (A) EGF expression: note the slight decline in the SMOF and OOFE groups; however, no changes were significant. (B) EGF expression: note the significant decline in the SMOF and OOFE groups. (C) Epiregulin expression: note the significant decline in the OOFE group. (D) TGF- $\alpha$ expression: note the significant decline in the OOFE group. EGF, epidermal growth factor; OOFE, 80\% olive oil-supplemented fat emulsion; PN, parenteral nutrition; SBFE, standard soybean-based fat emulsion; SMOF, combination of soybean oil, medium-chain triglycerides, olive oil, and fish oil emulsion; TGF- $\alpha$, transforming growth factor- $\alpha$.

improvement in lymphocyte function was identified. ${ }^{26}$ In another study between fish oil vs soybean oil, fish oil led to an increase in TH2-based cytokine formation within splenocytes. ${ }^{27}$ In a clinical study of preterm infants, a reduction of IL-6 was observed in patients fed olive oil vs soybean FE. ${ }^{28}$ Finally, human leukocytes showed a marked improvement in lymphocyte activation in olive oil FE exposed vs soybean FE cells. ${ }^{29}$ Together, this suggests improved immune function and a decline in proinflammatory cytokines with either olive oilor fish oil-based FEs. The precise mechanism by which the OOFE group had markedly improved survival is unknown. It is quite possible that this could be due to an effect of the unique antioxidant effect or differences in polyphenol composition. It is also possible that the reduced levels of proinflammatory cytokines in this lipid group may be a major contributory mechanism.

Barrier function has been shown to significantly decline with administration of $\mathrm{PN} .{ }^{11,12}$ The assessment of tight junction factors, occludin, ZO-1, claudin-2, and claudin-15, demonstrated some differences between the groups, with a moderate increase in occludin, claudin-2, and ZO-1 in the SBFE group and a decline in all FE groups for claudin-15. Interestingly, TER was noted to be significantly decreased in all FE groups, regardless of the mRNA changes in tight junction factors. This 
TNF- $\alpha$

A.

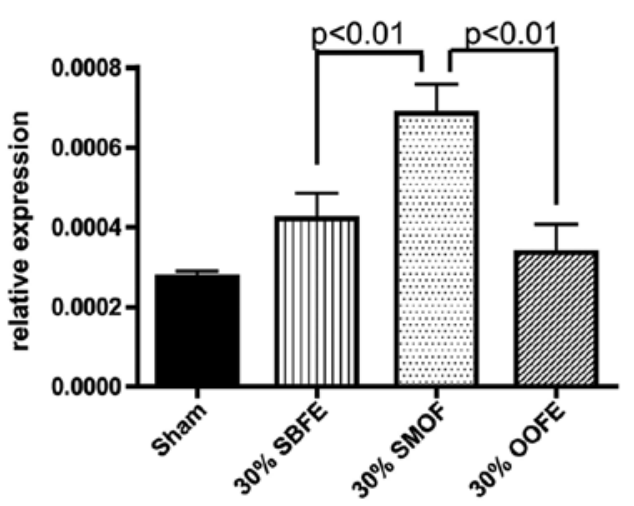

B.

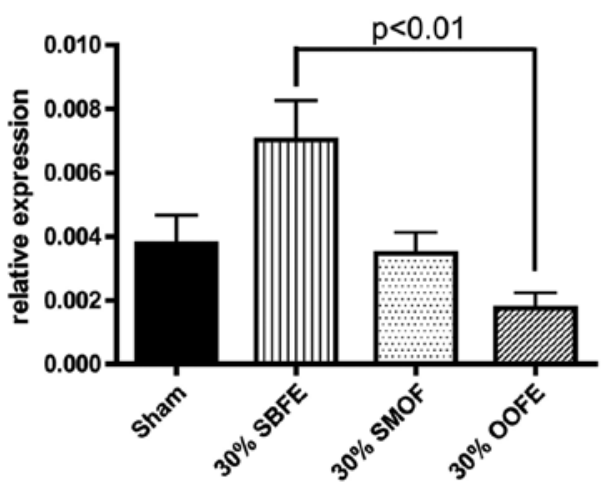

C.

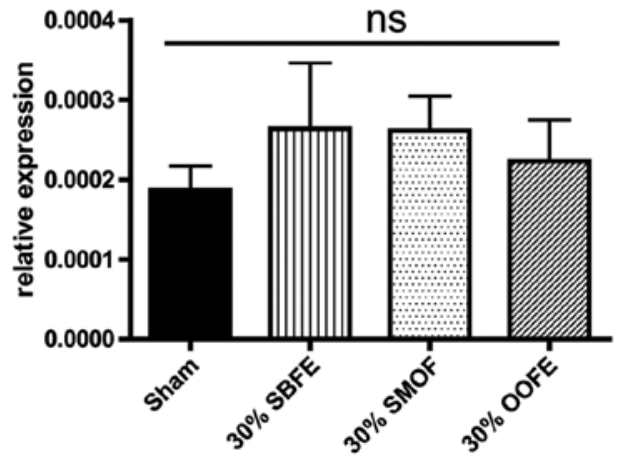

TNFR1

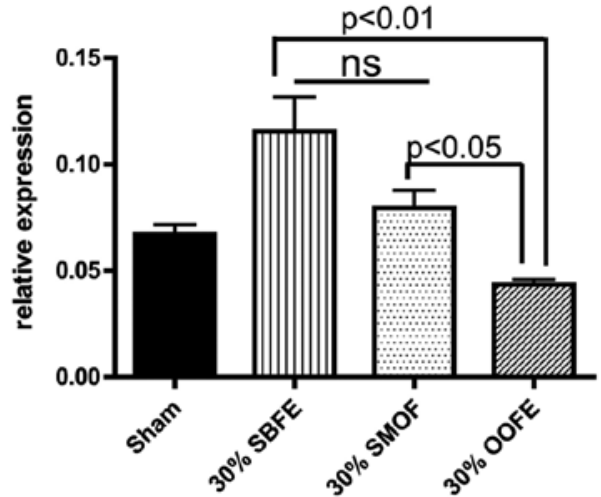

IL-6

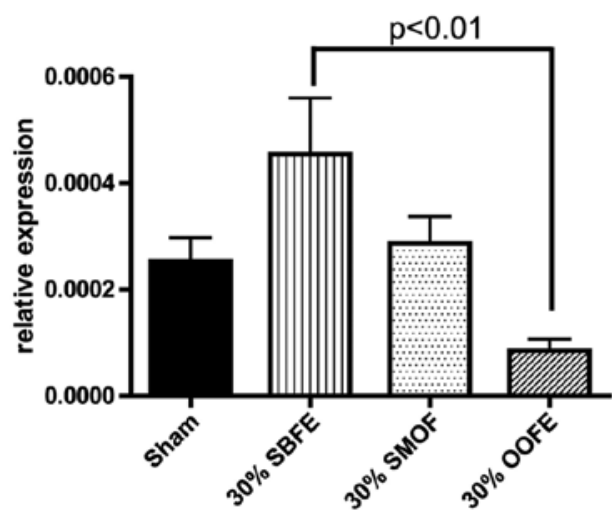

Toll like receptor 4 (TIr4)

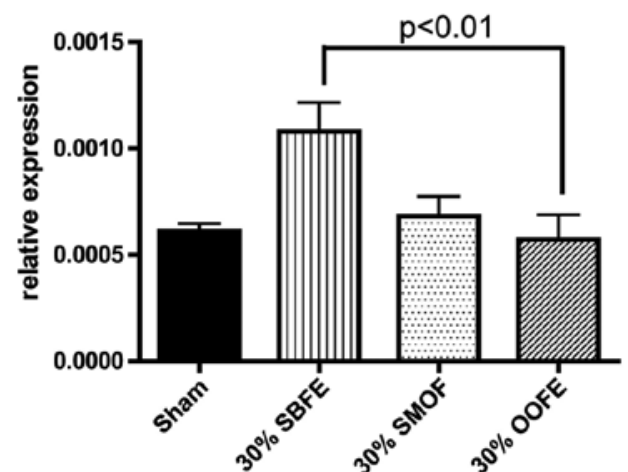

Figure 5. Proinflammatory cytokines and signaling receptors were increased with PN but downregulated with OOFE. Proinflammatory cytokines and the selected TNF- $\alpha$ receptor R1 (TNFR1) were measured with real-time polymerase chain reaction and corrected for expression by measuring $\beta$-actin messenger RNA. (A) TNF- $\alpha$ expression showed that the SMOF group had the highest levels of TNF- $\alpha$ $(P<.01)$ compared with either the SBFE or OOFE group. TNFR1 expression was highest in the SBFE group $(P<.01)$, with slightly reduced levels in the SMOF group and significantly lower levels in the OOFE group $(P<.01)$. (B) IL- 6 and IL- $1 \beta$ expression were both significantly increased in the SBFE group. Levels of these cytokines were reduced in the SMOF group to that of a similar range to sham mice, and levels were lowest in the OOFE group and declined below that of sham mice $(P<.01)$. (C) INF- $\gamma$ levels did not significantly change between study groups, whereas Tlr4 expression significantly increased in the SBFE group compared with sham mice and was significantly decreased in the OOFE group $(P<.01)$. IL, interleukin; INF- $\gamma$, interferon $\gamma$; OOFE, 80\% olive oil-supplemented fat emulsion; PN, parenteral nutrition; SBFE, standard soybean-based fat emulsion; SMOF, combination of soybean oil, medium-chain triglycerides, olive oil, and fish oil emulsion; TGF- $\alpha$, transforming growth factor- $\alpha$; Tlr4, Toll-like receptor 4. 
A.
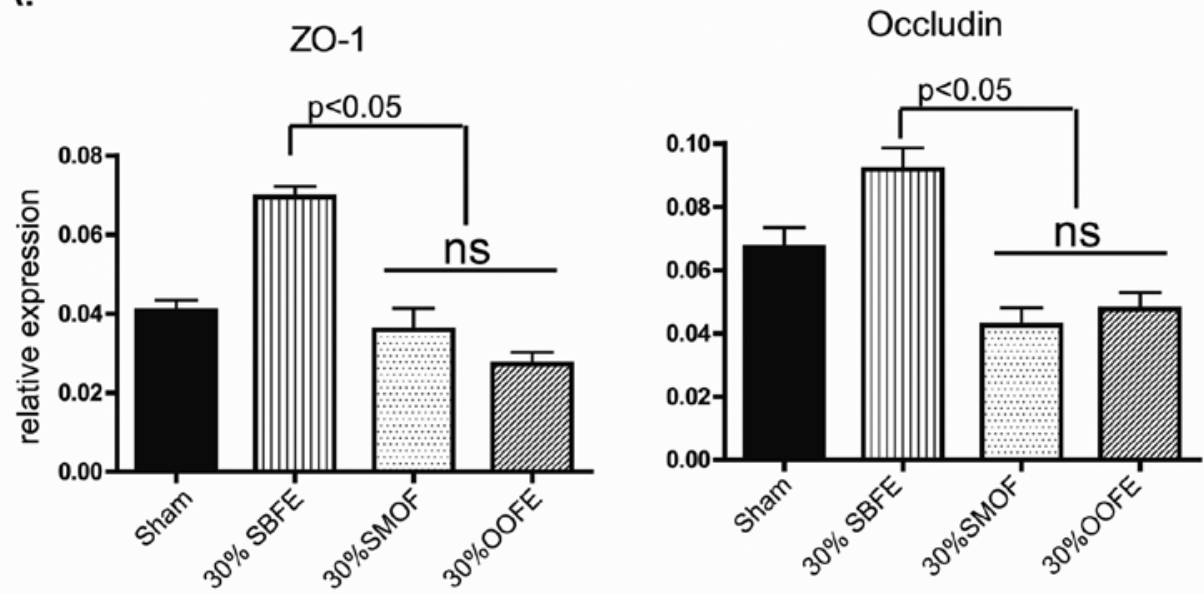

Claudin 15

B.

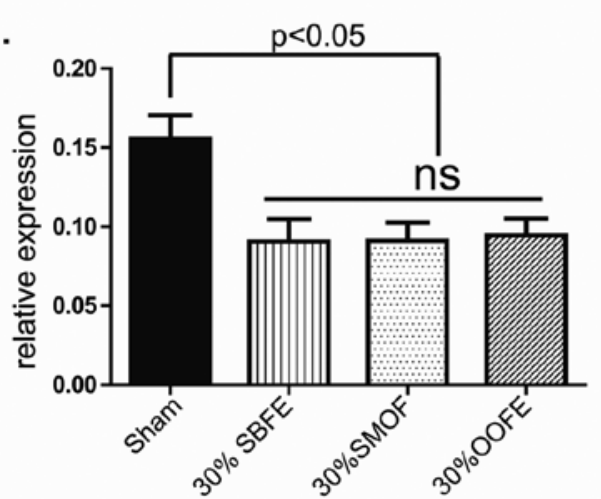

\section{Claudin2}

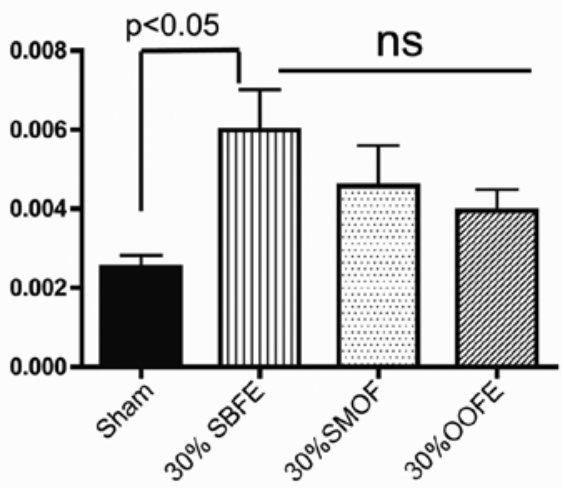

C.

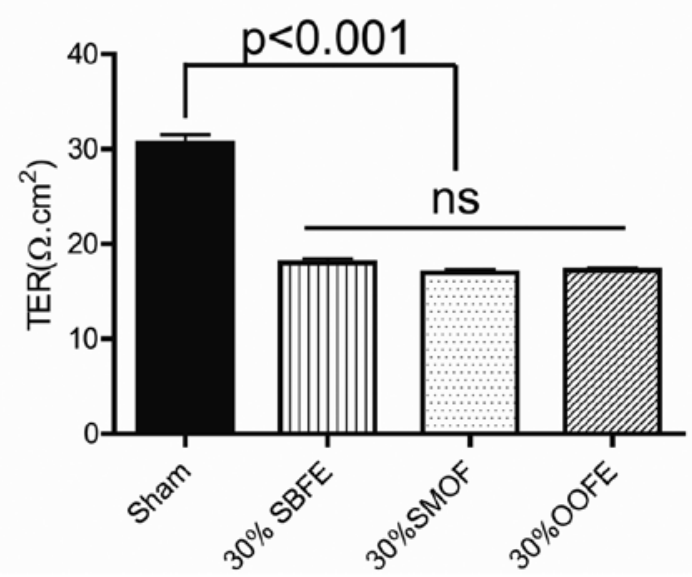

Figure 6. Jejunal barrier function was decreased with PN administration. EBF was measured by the abundance of selected tight junction factors at the mRNA level. EBF was also measured detecting TER in full-thickness jejunal tissue. (A) mRNA levels of tight junction factors ZO-1 and occludin rose modestly in the SBFE group and significantly declined in the SMOF and OOFE groups $(P<.05)$. (B) Claudin-2 mRNA abundance rose significantly in all PN groups, with the highest abundance noted in the SBFE group $(P<.05)$. Claudin-15 mRNA levels were significantly lower than the sham group in all FE study groups $(P<.05)$. (C) TER measures showed a significant decline in all FE study groups compared with the sham group $(P<.05)$. EBF, epithelial barrier function; FE, fat emulsion; mRNA, messenger RNA; OOFE, $80 \%$ olive oil-supplemented fat emulsion; PN, parenteral nutrition; SBFE, standard soybean-based fat emulsion; SMOF, combination of soybean oil, medium-chain triglycerides, olive oil, and fish oil emulsion; TER, transepithelial resistance. 
Table 2. Fatty Acid Content From Jejunal Mucosal Tissue From Mice.

\begin{tabular}{|c|c|c|c|c|}
\hline Fatty Acid & Sham & SBFE $30 \%$ & SMOF $30 \%$ & OOFE $30 \%$ \\
\hline $\begin{array}{l}\text { Total content of fatty acids from jejunal } \\
\text { mucosa, } \mathrm{mg} / \mathrm{g} \text { of mucosal tissue }\end{array}$ & $13.97 \pm 4.07(\mathrm{n}=6)$ & $17.60 \pm 7.67(\mathrm{n}=8)$ & $15.56 \pm 7.45(\mathrm{n}=8)$ & $15.70 \pm 2.83(n=8)$ \\
\hline \multicolumn{5}{|c|}{ Total content of fatty acids represented as the percent of all fatty acids, mean $\pm \mathrm{SD}$} \\
\hline C16:0 (palmitic acid) & $19.649 \pm 0.549$ & $15.025 \pm 5.828$ & $17.250 \pm 1.450$ & $18.027 \pm 1.070$ \\
\hline C18:0 (stearic acid) & $18.579 \pm 1.388$ & $20.020 \pm 2.261$ & $19.708 \pm 1.597$ & $18.172 \pm 1.733$ \\
\hline 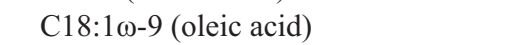 & $8.760 \pm 2.396$ & $9.051 \pm 4.611$ & $11.055 \pm 3.370$ & $12.135 \pm 2.383$ \\
\hline 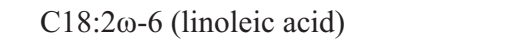 & $28.425 \pm 1.399$ & $24.762 \pm 3.306$ & $21.430 \pm 1.667$ & $20.238 \pm 1.758$ \\
\hline $\mathrm{C} 18: 3 \omega-3$ ( $\alpha$ linolenic acid $)$ & $0.485 \pm 0.261$ & $0.209 \pm 0.045$ & $0.202 \pm 0.214$ & $0.186 \pm 0.117$ \\
\hline 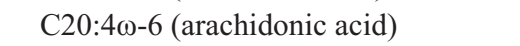 & $8.290 \pm 0.996$ & $13.549 \pm 1.924$ & $10.490 \pm 2.148$ & $13.582 \pm 2.899$ \\
\hline 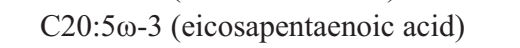 & $1.568 \pm 0.122$ & $0.616 \pm 0.290$ & $2.817 \pm 0.594$ & $0.582 \pm 0.182$ \\
\hline $\mathrm{C} 22: 5 \omega-3$ (docosapentaenoic acid) & $0.640 \pm 0.087$ & $0.391 \pm 0.083$ & $0.951 \pm 0.108$ & $0.360 \pm 0.049$ \\
\hline 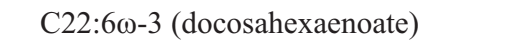 & $5.186 \pm 0.687$ & $5.945 \pm 0.947$ & $6.680 \pm 0.717$ & $6.562 \pm 0.464$ \\
\hline
\end{tabular}

Fatty Acid Sham vs Sham SBFE 30\% vs Sham SMOF $30 \%$ vs Sham OOFE $30 \%$ vs Sham

\begin{tabular}{|c|c|c|c|c|}
\hline \multicolumn{5}{|c|}{ Ratio of fatty acids compared with value in sham or enterally fed group } \\
\hline $\mathrm{C} 16: 0$ & 1 & 0.76 & 0.88 & 0.92 \\
\hline $\mathrm{C} 18: 0$ & 1 & 1.08 & 1.06 & 0.98 \\
\hline $\mathrm{C} 18: 1 \omega-9$ & 1 & 1.03 & 1.26 & 1.39 \\
\hline $\mathrm{C} 18: 2 \omega-6$ & 1 & 0.87 & 0.75 & 0.71 \\
\hline $\mathrm{C} 18: 3 \omega-3$ & 1 & 0.43 & 0.42 & 0.38 \\
\hline $\mathrm{C} 20: 4 \omega-6$ & 1 & 1.63 & 1.27 & 1.64 \\
\hline $\mathrm{C} 20: 5 \omega-3$ & 1 & 0.39 & 1.8 & 0.37 \\
\hline $\mathrm{C} 22: 5 \omega-3$ & 1 & 0.61 & 1.49 & 0.56 \\
\hline $\mathrm{C} 22: 6 \omega-3$ & 1 & 1.15 & 1.29 & 1.27 \\
\hline
\end{tabular}

OOFE, $80 \%$ olive oil-supplemented fat emulsion; SBFE, standard soybean-based fat emulsion; SMOF, combination of soybean oil, medium-chain triglycerides, olive oil, and fish oil emulsion.

is most likely due to the fact that EBF correlates better with the actual structural integrity of these factors, ${ }^{30}$ and these could all be disrupted with the measured increase in proinflammatory markers and/or loss of mucosal growth factors in the PN groups.

Mechanisms that might account for these differences are not known. One of the more intriguing findings was the marked decline in proinflammatory cytokines in the OOFE group, yet a marked decline in the abundance of several growth factors. It has been increasingly appreciated over the past 5 years that there is a tight transactivation between TNF- $\alpha$ and EGF. ${ }^{31}$ However, with PN administration, our group has previously reported an increase in TNF- $\alpha$ and a marked loss of EGF and several EGF family members. ${ }^{22}$ It is possible that the increased mRNA abundance of TNF- $\alpha$ in the SBFE and SMOF groups and loss of EGF in the OOFE group led to the uniform loss of EBF in all FE study groups.

The changes in mucosal FAs measured in the present study were interesting and may have significant physiologic correlates. For instance, the marked increase in AA in the OOFE group could result in enhanced protection of the host, despite the loss of EBF. Recently, enhancement of AA levels within the small bowel was shown to improve barrier function in an acute ischemia model in suckling piglets. ${ }^{32}$ In contrast, the increase in EPA in the SMOF group might help protect the intestine from the increase in proinflammatory cytokines, as EPA has also been shown to enhance $\mathrm{EBF}^{32}$

However, the combination of lipids and the precise mechanisms that have resulted in the present findings are complex and not fully determined in this study. It is also not clear how differing FAs may have affected the intestinal barrier function in our study. It has been reported that polyunsaturated FAs induce intracellular acidosis, which can decrease the intracellular adenosine triphosphate level and inhibit $\mathrm{Ca}^{+2}$-ATPase. The increase in the calcium level activates actomyosin contraction through the activation of cytoskeletal stabilization or through other processes leading to the opening of the tight junctions. ${ }^{33}$ Some investigators have shown that DHA and EPA have a positive effect on impaired EBF induced by IFN- $\gamma$ and TNF. ${ }^{34}$ However, this was not shown in the current study. Therefore, this suggests that other factors may contribute to changes in both EC proliferation and injury of barrier function in this PN model, and future studies will need to be done.

One important issue was that we selected a $30 \%$ lipid dose to make this most relevant to the clinical delivery of nutrition. This amount of lipid is higher than what most mouse diets 
consist of. Although it is possible that a mouse may be less tolerant of this higher lipid dose, we felt that the greatest influence on survival and the proinflammatory state was due to the type of lipid, rather than the absolute dose. Whether the present findings will translate to a clinical setting remains to be determined. As an example, a recent double-blind, randomized, clinical trial comparing soybean oil vs olive oil FE in an adult intensive care setting failed to demonstrate any differences in survival or a reduction in infectious complications. ${ }^{35}$ In contrast, a recent systematic review suggested that fish oil lipid emulsions resulted in improved infectious outcomes in surgical patients. ${ }^{36}$ Use of a combination FE, SMOF, led to similar if not superior results in a prospective controlled double-blind study ${ }^{37}$ Presently, only 1 class of IVFEs is available in the United States, with all being soybean-based products, whereas the other 2 FEs studied in this article are available in other regions of the world, including Europe, Asia, the Middle East, and South America. Based on the findings of this study and many other lines of animal and clinical studies, it is increasingly evident that newer forms of FE need to be studied and used in the United States. Such a position recently has been taken by the American Society for Parenteral and Enteral Nutrition, and it is hoped that such efforts will lead to improved nutrition care for those patients receiving PN. ${ }^{38}$ One limitation of this study was that we confined our expression of cytokine abundance and growth factors to the mRNA level. To take this to the protein level would far exceed the scope of the present study, but it will be important to carry this further in future investigations. Another limitation was that we focused on $30 \%$ of nutrients being delivered as an FE. Although this may be clinically relevant to humans, it is higher than what most rodents use on a daily basis. Thus, a perfect correlation with the use of this model is not completely possible.

The implications of this work are considerable in that the clinical application and selection of one type of IV lipid over another is not readily clear, and the insights provided here might help a clinician select one form over another or decide in which clinical setting a particular FE may be most appropriate. Clearly, these results are confined to the intestine, and a broader examination of the physiologic effects of these lipids will need to be performed.

\section{References}

1. Hippalgaonkar K, Majumdar S, Kansara V. Injectable lipid emulsionsadvancements, opportunities and challenges. AAPS PharmSciTech. 2010;11(4):1526-1540.

2. Iba T, Yagi Y, Kidokoro A, Ohno Y, Kaneshiro Y, Akiyama T. Total parenteral nutrition supplemented with medium-chain triacylglycerols prevents atrophy of the intestinal mucosa in septic rats. Nutrition. 1998;14(9):667-671.

3. Ulrich H, Pastores SM, Katz DP, Kvetan V. Parenteral use of mediumchain triglycerides: a reappraisal. Nutrition. 1996;12(4):231-238.

4. Gogos CA, Zoumbos N, Makri M, Kalfarentzos F. Medium- and longchain triglycerides have different effects on the synthesis of tumor necrosis factor by human mononuclear cells in patients under total parenteral nutrition. J Am Coll Nutr. 1994;13(1):40-44.
5. Waitzberg DL, Torrinhas RS. Fish oil lipid emulsions and immune response: what clinicians need to know. Nutr Clin Pract. 2009;24(4): 487-499.

6. Carpentier YA, Dupont IE. Advances in intravenous lipid emulsions World J Surg. 2000;24(12):1493-1497.

7. Waitzberg DL, Torrinhas RS, Jacintho TM. New parenteral lipid emulsions for clinical use. JPEN J Parenter Enteral Nutr. 2006;30(4):351-367.

8. Meisel JA, Le HD, de Meijer VE, et al. Comparison of 5 intravenous lipid emulsions and their effects on hepatic steatosis in a murine model. $J$ Pediatr Surg. 2011;46(4):666-673.

9. de Meijer VE, Gura KM, Le HD, Meisel JA, Puder M. Fish oil-based lipid emulsions prevent and reverse parenteral nutrition-associated liver disease: the Boston experience. JPEN J Parenter Enteral Nutr. 2009;33(5):541-547.

10. Feng Y, McDunn JE, Teitelbaum DH. Decreased phospho-Akt signaling in a mouse model of total parenteral nutrition: a potential mechanism for the development of intestinal mucosal atrophy. Am J Physiol Gastrointest Liver Physiol. 2010;298(6):G833-G841.

11. Yang H, Finaly R, Teitelbaum DH. Alteration in epithelial permeability and ion transport in a mouse model of total parenteral nutrition. Crit Care Med. 2003;31(4):1118-1125.

12. Yang H, Sun X, Haxhija EQ, Teitelbaum DH. Intestinal epithelial cellderived interleukin-7: a mechanism for the alteration of intraepithelial lymphocytes in a mouse model of total parenteral nutrition. Am J Physiol Gastrointest Liver Physiol. 2007;292(1):G84-G91.

13. Wildhaber BE, Yang H, Tazuke Y, Teitelbaum DH. Gene alteration of intestinal intraepithelial lymphocytes with administration of total parenteral nutrition. J Pediatr Surg. 2003;38(6):840-843.

14. Kiristioglu I, Teitelbaum DH. Alteration of the intestinal intraepithelial lymphocytes during total parenteral nutrition. J Surg Res. 1998;79(2): 91-96.

15. Sun X, Spencer AU, Yang H, Haxhija EQ, Teitelbaum DH. Impact of caloric intake on parenteral nutrition-associated intestinal morphology and mucosal barrier function. JPEN J Parenter Enteral Nutr. 2006;30(6): 474-479.

16. Nose K, Yang H, Sun $X$, et al. Glutamine prevents total parenteral nutrition-associated changes to intraepithelial lymphocyte phenotype and function: a potential mechanism for the preservation of epithelial barrier function. J Interferon Cytokine Res. 2010;30(2):67-80.

17. Feng Y, McDunn J, Teitelbaum D. Decreased phospho-Akt signaling in a mouse model of total parenteral nutrition (TPN): a potential mechanism for the development of intestinal mucosal atrophy. Gastroenterology. 2009;136(5)(suppl 1):A-96.

18. Yang H, Fan Y, Teitelbaum DH. Intraepithelial lymphocyte-derived interferon-gamma evokes enterocyte apoptosis with parenteral nutrition in mice. Am J Physiol Gastrointest Liver Physiol. 2003;284(4):G629-G637.

19. Feng Y, Sun X, Yang H, Teitelbaum DH. Dissociation of E-cadherin and beta-catenin in a mouse model of total parenteral nutrition: a mechanism for the loss of epithelial cell proliferation and villus atrophy. J Physiol. 2009;587(pt 3):641-654.

20. Xu Z, Harvey K, Pavlina T, Dutot G, Zaloga G, Siddiqui R. An improved method for determining medium- and long-chain FAMEs using gas chromatography. Lipids. 2010;45(2):199-208.

21. Sun $X$, Yang $H$, Nose $K$, et al. Decline in intestinal mucosal IL-10 expression and decreased intestinal barrier function in a mouse model of total parenteral nutrition. Am J Physiol Gastrointest Liver Physiol. 2008;294(1):G139-G147.

22. Feng Y, Teitelbaum DH. Epidermal growth factor/TNF-alpha transactivation modulates epithelial cell proliferation and apoptosis in a mouse model of parenteral nutrition. Am J Physiol Gastrointest Liver Physiol. 2012;302(2):G236-G249.

23. Yang H, Wildhaber B, Tazuke Y, Teitelbaum DH. 2002 Harry M. Vars Research Award. Keratinocyte growth factor stimulates the recovery of epithelial structure and function in a mouse model of total parenteral nutrition. JPEN J Parenter Enteral Nutr. 2002;26(6):333-341. 
24. Yang H, Wildhaber BE, Teitelbaum DH. 2003 Harry M. Vars Research Award. Keratinocyte growth factor improves epithelial function after massive small bowel resection. JPEN J Parenter Enteral Nutr. 2003;27(3):198-207.

25. Sun $\mathrm{X}$, Yang $\mathrm{H}$, Nose $\mathrm{K}$, et al. Decline in intestinal mucosal IL-10 expression and decreased intestinal barrier function in a mouse model of total parenteral nutrition. Am J Physiol Gastrointest Liver Physiol. 2008;294(1):G139-G147.

26. Lanza-Jacoby S, Flynn JT, Miller S. Parenteral supplementation with a fish-oil emulsion prolongs survival and improves rat lymphocyte function during sepsis. Nutrition. 2001;17(2):112-116.

27. Lin MT, Hsu CS, Yeh SL, et al. Effects of omega-3 fatty acids on leukocyte Th1/Th2 cytokine and integrin expression in rats with gut-derived sepsis. Nutrition. 2007;23(2):179-186.

28. Gawecka A, Michalkiewicz J, Kornacka MK, Luckiewicz B, Kubiszewska I. Immunologic properties differ in preterm infants fed olive oil vs soy-based lipid emulsions during parenteral nutrition. JPEN J Parenter Enteral Nutr. 2008;32(4):448-453

29. Granato D, Blum S, Rossle C, Le Boucher J, Malnoe A, Dutot G Effects of parenteral lipid emulsions with different fatty acid composition on immune cell functions in vitro. JPEN J Parenter Enteral Nutr. 2000;24(2):113-118.

30. Feng Y, Ralls MW, Xiao W, Miyasaka E, Herman RS, Teitelbaum DH. Loss of enteral nutrition in a mouse model results in intestinal epithelial barrier dysfunction. Ann N Y Acad Sci. 2012;1258:71-77.
31. Yamaoka T, Yan F, Cao $\mathrm{H}$, et al. Transactivation of EGF receptor and ErbB2 protects intestinal epithelial cells from TNF-induced apoptosis. Proc Natl Acad Sci U S A. 2008;105(33):11772-11777.

32. Jacobi SK, Moeser AJ, Corl BA, Harrell RJ, Blikslager AT, Odle J. Dietary long-chain PUFA enhance acute repair of ischemia-injured intestine of suckling pigs. J Nutr. 2012;142(7):1266-1271.

33. Hayashi M, Tomita M. Mechanistic analysis for drug permeation through intestinal membrane. Drug Metab Pharmacokinet. 2007;22(2): 67-77.

34. Li Q, Zhang Q, Wang M, Zhao S, Xu G, Li J. n-3 polyunsaturated fatty acids prevent disruption of epithelial barrier function induced by proinflammatory cytokines. Mol Immunol. 2008;45(5):1356-1365.

35. Umpierrez GE, Spiegelman R, Zhao V, et al. A double-blind, randomized clinical trial comparing soybean oil-based versus olive oil-based lipid emulsions in adult medical-surgical intensive care unit patients requiring parenteral nutrition. Crit Care Med. 2012;40(6):1792-1798.

36. Wei C, Hua J, Bin C, Klassen K. Impact of lipid emulsion containing fish oil on outcomes of surgical patients: systematic review of randomized controlled trials from Europe and Asia. Nutrition. 2010;26(5):474-481.

37. Klek S, Chambrier C, Singer $P$, et al. Four-week parenteral nutrition using a third generation lipid emulsion (SMOFlipid) - a double-blind, randomised, multicentre study in adults. Clin Nutr. 2013;32(2):224-231.

38. Vanek VW, Seidner DL, Allen P, et al. A.S.P.E.N. position paper: clinical role for alternative intravenous fat emulsions. Nutr Clin Pract. 2012;27(2):150-192. 